\title{
Fabrication of $\beta$-carotene nanoemulsion-based delivery systems using dual-channel microfluidization: Physical and chemical stability
}

\author{
Xiang Luo ${ }^{\mathrm{a}, \mathrm{b}}$, Yanyan Zhou ${ }^{\mathrm{c}}$, Long Bai ${ }^{\mathrm{b}}$, Fuguo Liu ${ }^{\mathrm{b}}$, Yihui Deng*,a, David \\ Julian McClements*,b
}

\author{
${ }^{a}$ College of Pharmacy, Shenyang Pharmaceutical University, 103 Wenhua Road, \\ Shenyang, Liaoning 110016, China \\ ${ }^{\mathrm{b}}$ Biopolymers and Colloids Laboratory, Department of Food Science, University of \\ Massachusetts, Amherst, MA 01003, USA \\ ${ }^{c}$ Research and Development Department, Zhejiang Medicine Co., Ltd Xinchang \\ Pharmaceutical Factory, 59 East Huancheng Road, Shaoxing, Zhejiang 312500, China
}

Journal: Journal of Colloid and Interface Science

Submitted: 2016

\section{Corresponding authors:}

Yihui Deng, E-mail address: dengyihui@ syphu.edu.cn; Telephone: +86 (0) 24 23986316

David Julian McClements, E-mail address: mcclements@foodsci.umass.edu;

Telephone: +1 4135451019 


\section{Abstract}

A considerable research effort is focused on developing effective delivery systems for hydrophobic nutraceuticals. $\beta$-carotene, a pro-vitamin A carotenoid, requires encapsulation to improve its water dispersibility and chemical stability in foods. In this study, $\beta$-carotene was encapsulated in oil-in-water nanoemulsions fabricated using high-pressure dual-channel microfluidization. Two types of natural emulsifier, quillaja saponins (Q-Naturale) and whey protein isolate (WPI), were capable of producing nanoemulsions $\left(d_{32}=0.14-0.16 \mu \mathrm{m}\right)$ using this novel homogenization method. The physical and chemical stability of these nanoemulsions were characterized during storage at neutral $\mathrm{pH}$ conditions at refrigeration $\left(4{ }^{\circ} \mathrm{C}\right)$, ambient $\left(25{ }^{\circ} \mathrm{C}\right)$, and elevated $\left(55{ }^{\circ} \mathrm{C}\right)$ temperatures. At 4 and $25{ }^{\circ} \mathrm{C}$, all nanoemulsions remained physically stable throughout 14 days storage, with little change in particle size or evidence of creaming. At $55{ }^{\circ} \mathrm{C}$, WPI nanoemulsions were also physically stable, but a small amount of droplet aggregation occurred in saponin nanoemulsions. The rate of $\beta$-carotene degradation increased with increasing storage temperature, but did not depend strongly on emulsifier type. This study showed that dual-channel microfluidization is an efficient method of continuously producing carotenoid-loaded nanoemulsions from natural emulsifiers. This knowledge may be useful for developing nutraceutical delivery systems for application within commercial food, beverage, and pharmaceutical products.

Keywords: nanoemulsion; dual-channel microfluidization; $\beta$-carotene; Q-Naturale; WPI; storage stability

\section{Introduction}

Carotenoids such as $\beta$-carotene are naturally produced at relatively high levels in 
many plants, animals, and microorganisms [1-3]. $\beta$-carotene has an intense red/orange/yellow color and may therefore be used as a natural pigment in foods. In addition, it has pro-vitamin A activity and may act as a nutraceutical that may inhibit the onset of cardiovascular diseases, eye diseases, certain cancers, and various other chronic diseases [4-5]. There is therefore considerable interest in incorporating $\beta$-carotene into foods and beverages as a natural pigment or nutraceutical agent [6]. However, $\beta$-carotene is a long hydrophobic molecule with a high melting point, which leads to an extremely low water-solubility and only a limited oil-solubility at room temperature. For this reason, $\beta$-carotene is difficult to incorporate into many aqueous-based foods, and tends to have a low and variable oral bioavailability [7]. Moreover, $\beta$-carotene is a polyunsaturated molecule that is highly susceptible to chemical degradation during food production, transport, and storage [8-10]. Consequently, it is necessary to develop an effective $\beta$-carotene delivery system to overcome these technical problems.

Previous researchers have been reported that $\beta$-carotene is primarily absorbed by epithelium cells due to passive diffusion after being incorporated into the mixed micelles formed in the gastrointestinal tract (GIT) after the digestion of dietary fats [11-12]. Based on this mechanism, nanoemulsion-based delivery systems have been proposed as one of the most convenient and effective methods of integrating $\beta$-carotene into foods $[10,13]$. $\beta$-carotene-enriched nanoemulsions can be fabricated by dissolving the carotenoid within a carrier oil, and then homogenizing this oil phase with an aqueous phase containing a water-soluble emulsifier. Nanoemulsions have a number of advantages over other types of delivery system for this purpose. First, they can be loaded with much more $\beta$-carotene than simple aqueous solutions [14]. Second, they are typically much more stable than conventional emulsions because the small particle size reduces the tendency for droplet aggregation and creaming to occur [15]. Third, nanoemulsions can rapidly and continuously be produced using various homogenization technologies $[10,13]$. 
Finally, the small size of the particles in nanoemulsions can increase the bioavailability of encapsulated hydrophobic nutraceuticals due to the more rapid and complete digestion of the lipid phase [16]. Previous research has shown that various approaches can be utilized to improve the physical and chemical stability of lipophilic bioactive components encapsulated in nanoemulsions, including metal ion chelation, antioxidant addition, surface potential adjustment, particle size control, and environmental regulation [17-18]. For example, in a recent study, the rate of $\beta$-carotene degradation was shown to be influenced by emulsifier type, storage temperature, and $\mathrm{pH}[9]$.

In this study, two natural "label-friendly" emulsifiers were used to form $\beta$-carotene-fortified nanoemulsions because there is increasing interest from consumers in "clean-label" food and beverage products: whey protein isolate (WPI) and quillaja saponins (Q-Naturale). WPI is isolated from bovine milk and is commonly used as an emulsifier to form and stabilize oil-in-water emulsions in the food industry [19]. Quillaja saponins are isolated from the Quillaja saponaria Molina tree and have also been shown to be effective emulsifiers in oil-in-water emulsions [20]. The nanoemulsions were fabricated using a relatively novel high-pressure dual-channel microfluidization method, which can be used to create nanoemulsions in a single step. In this method, an oil phase and an aqueous phase are fed through separate input channels that are made to impinge upon each other, and so there is no requirement for an initial pre-mixing stage. Dual channel microfluidization is therefore capable of saving time, energy, and costs, as well as reducing waste [21].

The objective of the present study was to compare the physical and chemical stability of $\beta$-carotene-loaded nanoemulsions fabricated by dual-channel microfluidization using either WPI or Q-Naturale as emulsifier. The effects of emulsifier concentration and homogenization pressure on nanoemulsion properties were investigated to better understand the difference in performance of these two natural 
emulsifiers. The information obtained from this study should facilitate the formation of nanoemulsion-based delivery systems for applications within food, beverage, and pharmaceutical products.

\section{Experimental}

\subsection{Materials}

Whey protein isolate (WPI) was purchased from Davisco Foods International Inc. (Le Sueur MN), which was reported to contain 97.9 wt.\% protein and 0.2 wt.\% fat. Quillaja saponin (Q-Naturale $\left.{ }_{\circledast} 200\right)$ was kindly provided by Ingredion Inc. (Westchester, IL). Corn oil (Mazola) was purchased from a local supermarket. $\beta$-carotene, sodium phosphate monobasic and disodium hydrogen phosphate were provided by Sigma-Aldrich Co. (St. Louis, MO). All chemicals used were analytical grade. Double distilled water (Milli-Q) was used to prepare all solutions.

\subsection{Preparation of $\beta$-carotene-loaded emulsions}

An aqueous phase was prepared by weighing an amount of WPI or Q-Naturale (0.1-3 wt $\%$ ) into a buffer solution (5 mM phosphate buffer, $\mathrm{pH} 7.0)$ and then stirring for 2 $\mathrm{h}$ to ensure dissolution. The resulting emulsifier solutions were then stored overnight at $4{ }^{\circ} \mathrm{C}$.

An oil phase was prepared by dispersing $0.1 \%(\mathrm{w} / \mathrm{w})$ of $\beta$-carotene in corn oil with heating $\left(55^{\circ} \mathrm{C}, 10 \mathrm{~min}\right)$ and then ultrasonic processing $(2 \mathrm{~min})$ at a frequency of $40 \mathrm{kHz}$., then stirring at ambient temperature for about $2 \mathrm{~h}$ to ensure full dissolution.

The $\beta$-carotene-loaded oil-in-water nanoemulsions were prepared by dual-channel microfluidizer (Microfluidics PureNano, Newton, MA, USA), as described in our

previous work [21]. In brief, the nanoemulsions were prepared by homogenizing $10 \%$ 
w/w oil phase with $90 \% \mathrm{w} / \mathrm{w}$ aqueous phase. Simultaneously, the oil phase and the aqueous phase were fed into the dual-channel microfluidizer through two separate glass reservoirs, and then made to impinge upon each other under high pressure (9-19 kpsi; 62-131 MPa).

\subsection{Determination of particle size and charge}

The mean droplet diameter and particle size distribution of $\beta$-carotene-loaded nanoemulsions were measured using static light scattering (Mastersizer 2000, Malvern Instruments Ltd., Malvern, Worcestershire, UK). Emulsions were diluted with the same aqueous phase buffer used in emulsion preparation to prevent multiple scattering effects. The droplet diameter of each sample is reported as the surface-weighted $\left(d_{32}\right)$ mean diameter:

$$
d_{32}=\sum n_{i} d_{i}^{3} / \sum n_{i} d_{i}^{2}
$$

The electrical surface potential ( $\zeta$-potential) of the droplets was measured using a Zetasizer Nano-ZS90 (Malvern Instruments, Worcestershire, UK). Samples were diluted 100 -fold with $5 \mathrm{mM}$ phosphate buffer $(\mathrm{pH} \mathrm{7)}$ ) and then transferred into a capillary test tube for measurement. Every sample was equilibrated for 60 second before data were collected using the Smoluchowski equation to convert the measured mobilities into $\zeta$-potential values.

\subsection{Microstructure analysis}

The microstructure of the samples was characterized using optical and confocal fluorescence microscopy (Nikon DEclipse C1 80i, Nikon, Melville, NY, USA), with a 10 $\times$ eyepiece and a $60 \times$ objective lens. For confocal microscopy, all the samples were dyed with Nile red (1 mg/mL ethanol), which is a hydrophobic fluorescent dye dissolved in 
ethanol. The excitation and emission spectrum for Nile Red were $543 \mathrm{~nm}$ and $605 \mathrm{~nm}$, respectively. All images were taken and processed using the instrument software program (EZ-CS1 version 3.8, Nikon, Melville, NY).

\subsection{Measurement of chemical degradation of $\beta$-carotene in emulsion}

The chemical degradation of $\beta$-carotene in the emulsions was measured using two different approaches: pigment extraction and colorimetry.

\section{Pigment extraction:}

For pigment extraction, the $\beta$-carotene was extracted form the nanoemulsions using an organic solvent, and then quantified using spectrophotometry. $3 \mathrm{ml}$ of $\beta$-carotene-loaded emulsions were mixed with $3 \mathrm{ml}$ of chloroform in a test tube, vortexed at high speed for $4 \mathrm{~min}$, and then centrifuged at $3000 \mathrm{rpm}$ for $15 \mathrm{~min}$ at $25{ }^{\circ} \mathrm{C}$. The transparent lower chloroform layer containing the $\beta$-carotene was transferred into a cuvette, and the absorbance was analyzed by a UV-visible spectrophotometer at $450 \mathrm{~nm}$ (Ultrospec 3000 Pro, Biochrom Ltd., Cambridge, UK). The concentration of $\beta$-carotene was calculated from the measured absorbance using a standard curve.

\section{Colorimetry:}

The colorimetric method is based on one described previously, which analyzes changes in the color coordinates of intact nanoemulsions during storage [9]. An aliquot $(10 \mathrm{ml})$ of nanoemulsion was transferred into a transparent cuvette, and then an instrumental colorimeter (ColorMunki, X-Rite, Grand Rapids, MI) was used to measure the tristimulus color coordinates $\left(\mathrm{L}^{*}, \mathrm{a}^{*}, \mathrm{~b}^{*}\right)$ based on the reflectance spectra. $\mathrm{L}^{*}$ indicates darkness or lightness and ranges from black (0) to white (100); $+\mathrm{a}^{*}$ is the red direction, $-a^{*}$ is the green direction, $+b^{*}$ is the yellow direction, and $-b^{*}$ is the blue direction. Chroma is the saturation or vividness of color [22]. The total color difference $\left(\Delta \mathrm{E}^{*}\right)$ of the nanoemulsions was calculated from the tristimulus values to provide a single 
measure of the total color change [23]:

$$
\Delta E^{*}=\left(\left(L^{*}-L_{0}^{*}\right)^{2}+\left(a^{*}-a_{0}^{*}\right)^{2}+\left(b^{*}-b_{0}^{*}\right)^{2}\right)^{1 / 2}
$$

Here $L^{*}, a^{*}$ and $b^{*}$ represent as color coordinates of the nanoemulsion at storage time t, and $L_{0}^{*}, a_{0}^{*}$, and $b_{0}^{*}$ are initial values.

\subsection{Effect of storage temperature on physical \& chemical stability of nanoemulsions}

To investigate the physical and chemical stability of $\beta$-carotene-loaded nanoemulsions at different storage temperatures, $15 \mathrm{ml}$ of samples were dispensed into 3 test tubes, then stored at 4,25 and $55{ }^{\circ} \mathrm{C}$ without light for 14 days. The samples were measured once every 2 days.

\subsection{Statistical analysis}

All data are shown as the mean \pm standard deviation (SD) of measurements made on at least two freshly prepared samples. Statistical comparisons were performed using Student's t-test with SPSS 16 software. $P$-values lower than 0.05 were considered to be significant.

\section{Results and discussion}

\subsection{Fabrication and characterization of nanoemulsion}

One of the main objectives of this study was to determine whether dual-channel microfluidization could be utilized to fabricate $\beta$-carotene-loaded nanoemulsions using natural emulsifiers. The dual-channel microfluidization method has some potential advantages over conventional homogenization methods for this purpose [21]. First, nanoemulsions can rapidly be formed using a continuous process that involves feeding 
separate oil and water phases into the homogenizer. Second, there is no need to form an emulsion premix or to carry out multiple passes through the homogenizer, which could save time, energy, and cost during the manufacturing process.

\subsubsection{Influence of emulsifier concentration on particle size}

Initially, the impact of emulsifier concentration on the characteristics of the droplets in $10 \mathrm{wt} \%$ corn oil-in-water emulsions produced using the dual-channel microfluidizer under fixed homogenization conditions (13 k or $90 \mathrm{MPa}, 1$ pass) was studied (Fig.1). There was a significant decrease in mean droplet diameter $\left(d_{32}\right)$ with increasing emulsifier concentration from 0.1 to $0.5 \mathrm{wt} \%$. This effect can be attributed to two major factors: (i) a greater amount of oil-water interfacial area can be stabilized in at higher emulsifier levels; (ii) faster coverage of the droplet surfaces reduces droplet coalescence within the homogenizer at higher emulsifier concentrations [24]. Above 0.5 wt\% emulsifier, the mean droplet diameter and particle size distribution did not change appreciably as the emulsifier level was increased further, which can be attributed to the fact that the droplet size was mainly limited by the maximum disruptive energy generated by the homogenizer rather than the emulsifier concentration [25]. The emulsions had monomodal particle size distributions at high emulsifier levels, which is important for many commercial applications. Similar general trends were observed for both types of emulsifier, but Q-Naturale was more effective at reducing the droplet size when used at the lowest emulsifier concentration $(0.1 \mathrm{wt} \%)$. At higher emulsifier levels, nanoemulsions $(d<200 \mathrm{~nm})$ could be formed using both types of emulsifier. 

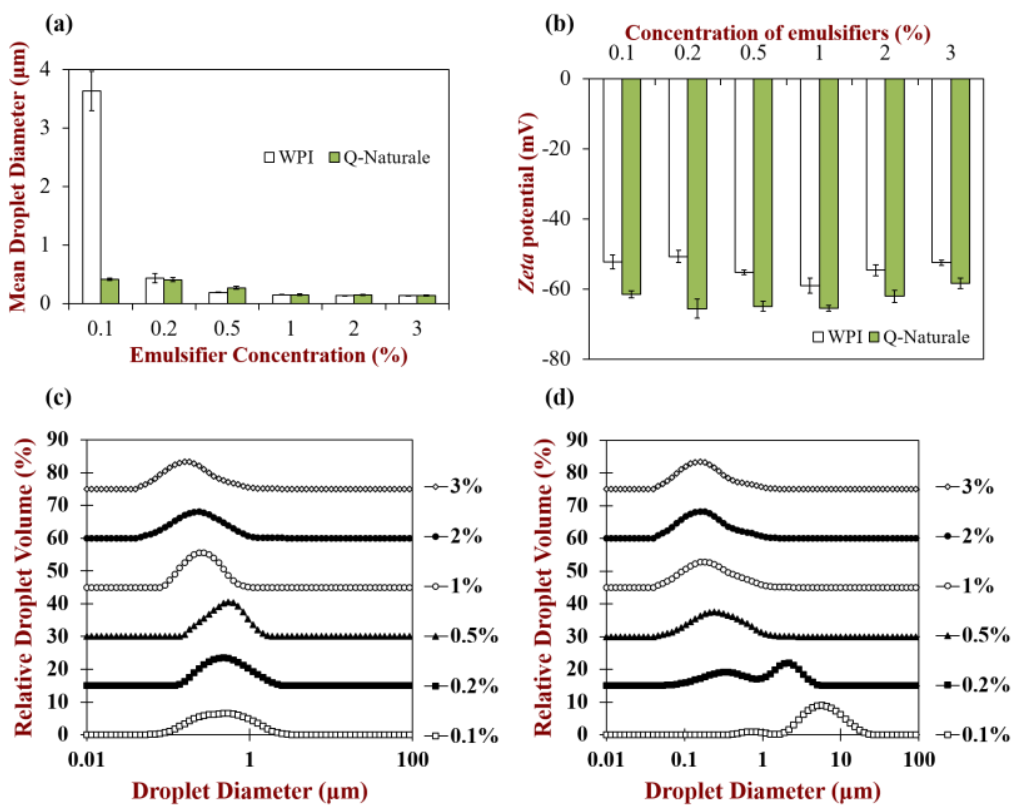

Fig. 1. Influence of emulsifier concentration on the mean droplets diameter $\left(d_{32}\right), \zeta$-potential and particle size distribution of $\beta$-carotene-loaded emulsions prepared by dual-channel microfluidization under fixed homogenization conditions (13 kpsi or $90 \mathrm{MPa}, 1$ pass).

All the emulsions contained negatively charged droplets at the neutral $\mathrm{pH}$ conditions used to form them, with Q-Naturale giving slightly more negative droplets than WPI (Fig.1b). The negative charge on the Q-Naturale-coated droplets is due to the ionization of carboxyl groups on the quillaja saponin molecules, whereas the negative charge on the WPI-coated droplets is due to the fact that there are more negatively charged carboxyl groups than positively charged amino groups above the protein's isoelectric point. The relatively high magnitude of the $\zeta$-potential on the oil droplets suggests that both types of emulsion would be stable to aggregation at this $\mathrm{pH}$ due to a strong electrostatic repulsion acting between the droplets [26].

In the remainder of the studies, $1 \mathrm{wt} \%$ emulsifier was used to fabricate the $\beta$-carotene-loaded nanoemulsions since it gave small droplets with a monomodal particle size distribution and high electrical charge. 


\subsubsection{Influence of homogenization pressure on particle size}

The size of the droplets in oil-in-water emulsions produced by high-pressure homogenization depends on the operating pressure as this determines the maximum disruptive energy available to breakup the droplets [27-28]. We therefore investigated the effect of homogenization pressure on the characteristics of the oil droplets produced using the two types of natural emulsifier used in this study (Fig.2). The influence of homogenization pressure on the mean droplet diameter, particle size distribution and $\zeta$-potential of droplets formed by dual-channel microfluidization was determined for 10 wt $\%$ oil-in-water systems stabilized by $1 \mathrm{wt} \%$ Q-Naturale or WPI. There was a decrease in mean particle diameter with increasing operating pressure for both emulsifiers (Fig. 2a.), which can be attributed to an increase in the magnitude of the disruptive energy generated by the homogenizer at higher pressures [29]. Over the range of pressures used ( 9 to $19 \mathrm{kpsi}$ or 62 to $131 \mathrm{MPa}$ ), the mean droplet diameter in the Q-Naturale emulsions decreased from 204 to $143 \mathrm{~nm}$, whereas that in the WPI emulsions decreased from 170 to $136 \mathrm{~nm}$. Interestingly, the mean droplet diameter was fairly similar for both types of emulsifier when the homogenization pressure exceeded about $15 \mathrm{kpsi}$ (103 MPa).

There was a linear decrease in the logarithm of the mean droplet diameter with the logarithm of the operating pressure for the two nanoemulsions (Fig. 2a), which is consistent with previous studies that indicate there is a linear relationship as long as there is sufficient emulsifier present [30]. The slope determined from the $\log _{10}(d)$ versus $\log _{10}(P)$ plots was around -0.51 when Q-Naturale was used, and around -0.26 when WPI was used. Values of the slope around -0.6 to -0.8 have previously been reported for high-pressure homogenizers when the fluid flow conditions are primarily turbulent-inertial [31]. This suggests that either droplet breakup was not primarily due to turbulent-inertial forces in the dual-channel microfluidizer used in this study. The 
manufacturers of the device report that the shear forces generated within a dual-channel microfluidizer are around an order of magnitude greater than those caused by other types of high-pressure homogenizer, which might explain this result.
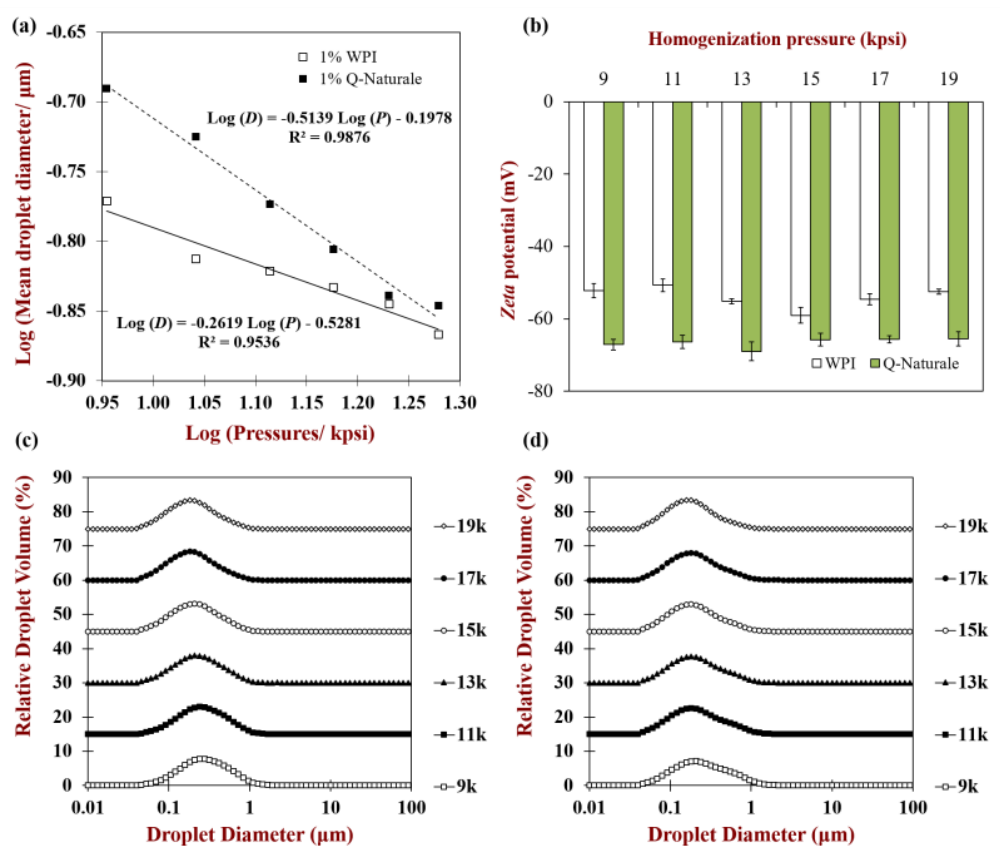

Fig.2. Influence of homogenization pressure on the mean particle diameter $\left(d_{32}\right)$ and size distribution of $\beta$-carotene-loaded emulsions prepared by dual-channel methods.

The impact of operating pressure on the particle size distributions of the nanoemulsions prepared using the two different natural emulsifiers was also recorded (Figs. 2c and 2d). For both emulsifiers, the width of the particle size distribution decreased with increasing homogenization pressure, and the particle size distribution was monomodal at all pressures studied. This data indicates droplet disruption was efficient when the aqueous phase and oil phase were only passed through this homogenizer once. These results suggest that the dual-channel microfluidizer is highly efficient at forming small droplets. 


\subsection{Influence of storage temperature on physical and chemical stability of nanoemulsions}

Commercially, nutraceutical delivery systems may be exposed to a range of different temperatures during their storage and transport. For this reason, the effect of storage temperature on the physical and chemical stability of $\beta$-carotene-enriched nanoemulsions was examined. The nanoemulsions were stored at 4,25 and $55{ }^{\circ} \mathrm{C}$ to simulate refrigeration, ambient, and elevated storage temperatures, respectively.

\subsubsection{Physical stability of nanoemulsions}

Initially, the mean particle diameters of the nanoemulsions stabilized by Q-Naturale or WPI were relatively small: $d_{32}=0.14-0.16 \mu \mathrm{m}$ (Figs. $3 \mathrm{a}$ and $3 \mathrm{c}$ ). In addition, these nanoemulsions had monomodal particle size distributions (Figs. 2c and 2d) and homogeneous microstructures (Fig. 4). These results confirm that the dual-channel microfluidization method was efficient at fabricating stable nanoemulsions. During storage, the Q-Naturale stabilized emulsions did not exhibit any significant droplet aggregation when incubated at 4 or $25{ }^{\circ} \mathrm{C}$ for 14 days, but there was a significant $(p<0.05)$ increase in mean particle diameter when they were stored at $55{ }^{\circ} \mathrm{C}$ (Fig. 3a). There was no evidence of creaming in any of the nanoemulsions during storage, which can be attributed to the fact that the droplets remained relatively small throughout storage $\left(d_{32}<\right.$ $0.16 \mu \mathrm{m})$. Previous researchers have also reported that Q-Naturale-coated oil droplets may flocculate or coalesce when stored at elevated temperatures for prolonged periods [32-33]. However, the $\zeta$-potential of these nanoemulsions did not change significantly throughout storage at any temperature (Fig.3b), which suggested that Q-Naturale remained at the droplet surfaces and did undergo any chemical reactions that altered its charge characteristics during storage. 
The mean particle diameter and $\zeta$-potential of the WPI-coated oil droplets did not change significantly during storage at any temperature, and there was no evidence of creaming, which can again be attributed to the relatively small droplet size. There was also no obvious change in the microstructure of any of the nanoemulsions during storage (Fig. 4), which was probably because the particle dimensions changed by less than $10 \mathrm{~nm}$ and was therefore undetectable by optical microscopy. These results suggest that nanoemulsions stabilized by Q-Naturale may be unstable to long-term storage at elevated temperatures, but that they are stable when stored at room temperature or below.
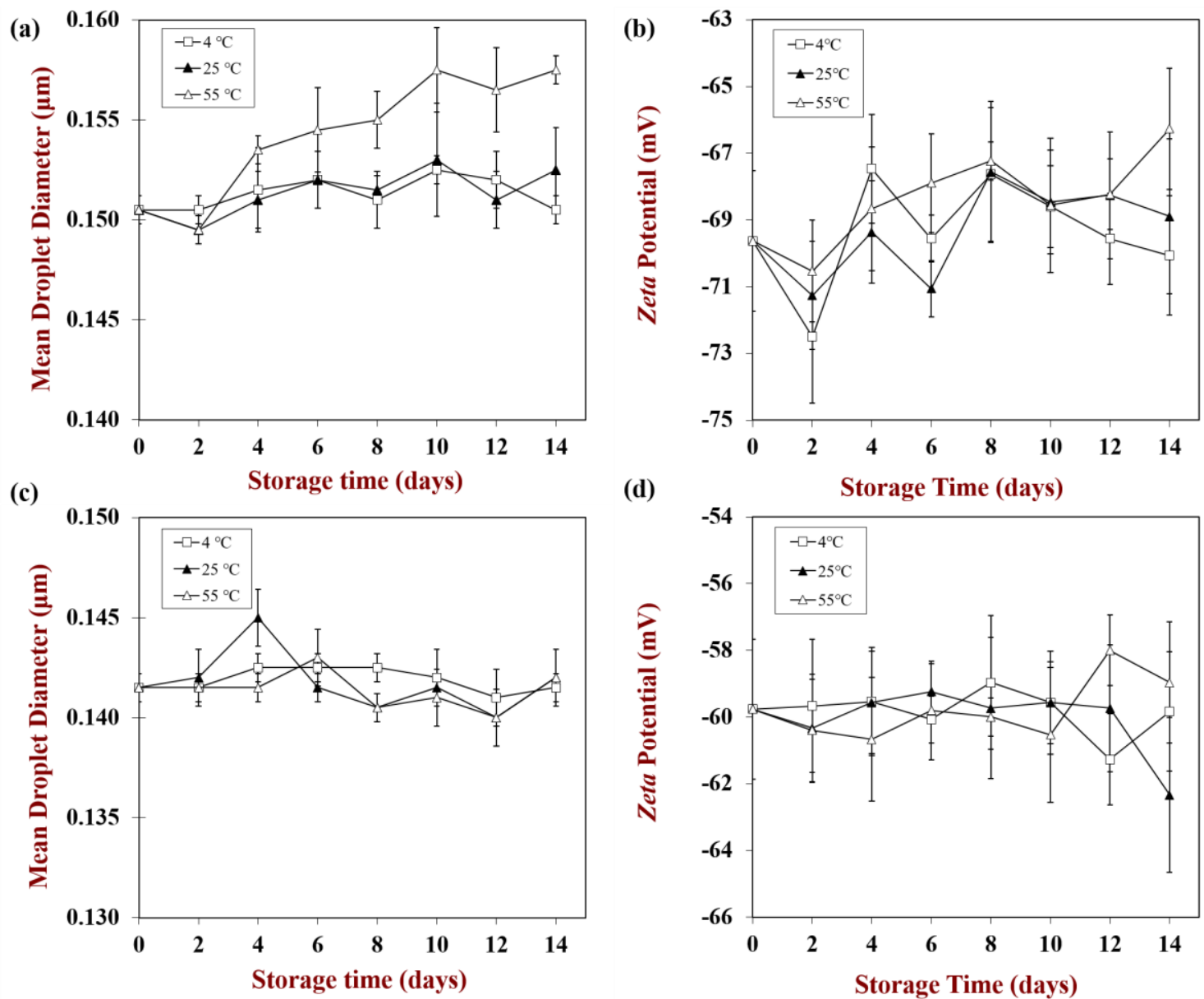

Fig.3. Influence of storage temperature on size in $\beta$-carotene-loaded nanoemulsions stabilized by Q-Naturale (a, b) or WPI (c, d) $(0.05 \% \beta$-carotene, $10 \%$ cron oil, $0.02 \%$ sodium azide, $5 \mathrm{mM}$ phosphate buffer, $\mathrm{pH} 7)$. 


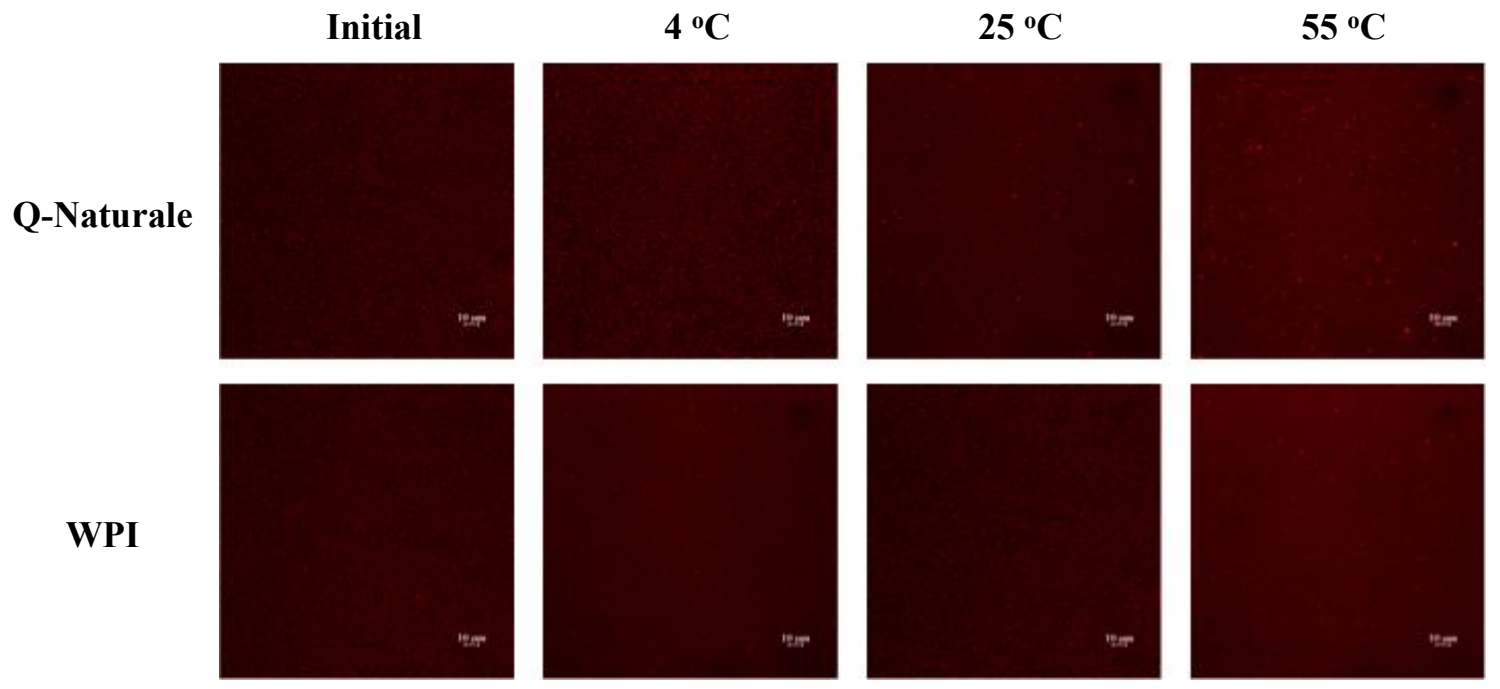

Fig.4. Influence of storage temperature on microstructure (determined by confocal fluorescence microscopy) of $\beta$-carotene-loaded nanoemulsions stabilized by Q-Naturale or WPI. The scale bars represent a length of $10 \mathrm{~nm}$, and the red regions represent lipids.

\subsubsection{Chemical stability of nanoemulsions}

The orange-red color of $\beta$-carotene becomes less intense when it undergoes chemical degradation [8]. Therefore, the chemical stability of $\beta$-carotene can be detected directly using solvent extraction methods or indirectly by measuring color fading of nanoemulsions using non-destructive colorimetry methods. Initially, colorimetry was used to measure the chemical stability of $\beta$-carotene in nanoemulsions stabilized by Q-Naturale or WPI (Fig. 5). In general, the lightness (L*) and color intensity ( $\mathrm{a}^{*}$ and $\mathrm{b}^{*}$ ) of the nanoemulsions progressively decreased during storage. These tristimulus color coordinates were then used to calculate the change in the total color difference $\left(\Delta \mathrm{E}^{*}\right)$ during storage. The rate of color fading increased as the storage temperature increased, with the most dramatic effect occurring between 25 and $55{ }^{\circ} \mathrm{C}$ (Fig. 5). These results 
show that the $\beta$-carotene-loaded in nanoemulsions are extremely unstable to chemical degradation when incubated at elevated temperatures. When the storage temperature was decreased from 25 to $4{ }^{\circ} \mathrm{C}$, there was a significant $(p<0.05)$ decrease in the rate of color fading in the Q-Naturale-stabilized nanoemulsions, but no significant difference for the WPI-stabilized nanoemulsions. Overall, the total amount of color fading was fairly similar for both types of nanoemulsions (Fig. 5). For example, the $\Delta \mathrm{E}^{*}$ values were 0.5 , 2.2 and 7.2 for the Q-Naturale systems and 1.2, 1.6 and 6.7 for the WPI systems after 14 days storage at 4,25 , and $55^{\circ} \mathrm{C}$, respectively.
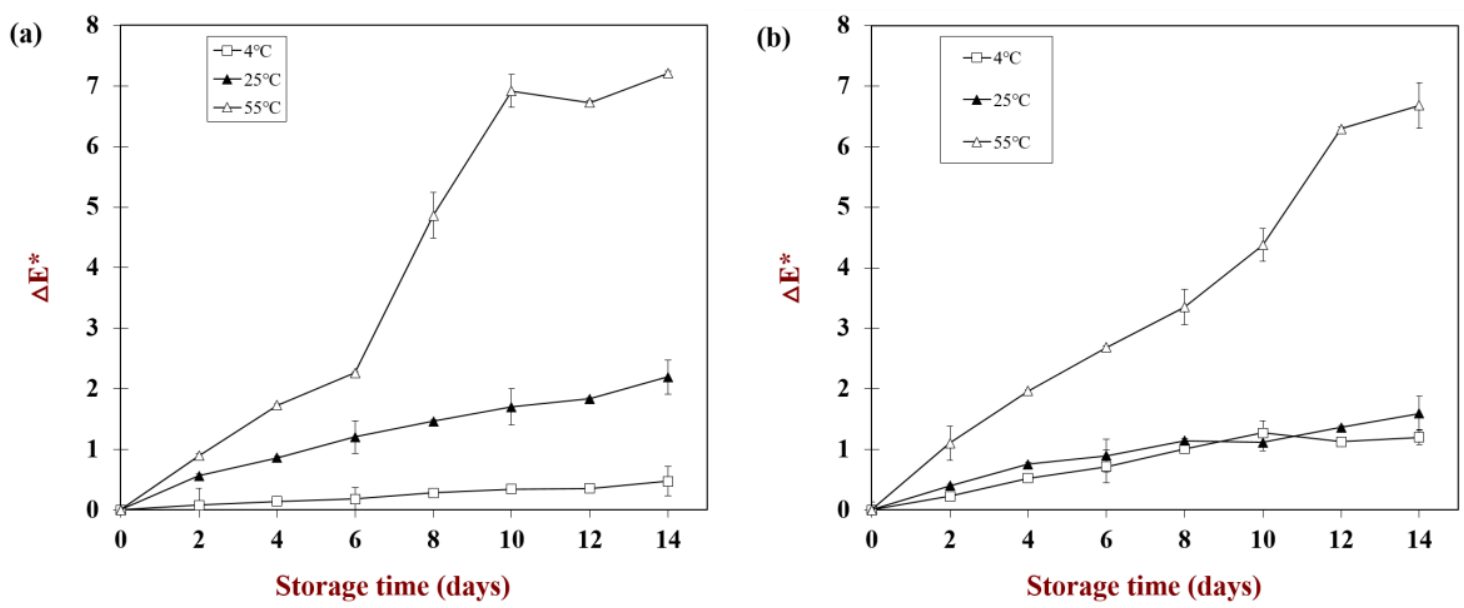

Fig.5. Influence of storage temperature on total colour change $\left(\Delta \mathrm{E}^{*}\right)$ of $\beta$-carotene-loaded nanoemulsions stabilized by (a) Q-Naturale or (b) WPI (0.05\% $\beta$-carotene, $10 \%$ cron oil, $0.02 \%$ sodium azide, $5 \mathrm{mM}$ phosphate buffer, $\mathrm{pH} 7)$.

A solvent extraction method was also used to measure the $\beta$-carotene concentration remaining in the nanoemulsions during storage at different temperatures (Fig. 6). The results obtained using this method were in relatively good agreement with those obtained using the colorimetry method. For the Q-Naturale-stabilized nanoemulsions, the rate of carotenoid degradation increased with increasing temperature, particularly between 25 and $55{ }^{\circ} \mathrm{C}$. For the WPI-stabilized systems, the rate of carotenoid degradation was much faster at $55{ }^{\circ} \mathrm{C}$ than at the two lower temperatures. As a result, there were appreciable 
differences in the amount of carotenoids remaining in the delivery systems at the end of the incubation period. After 14 days storage at 4,25 , and $55{ }^{\circ} \mathrm{C}$, the $\beta$-carotene concentration decreased from an initial value of $548 \mu \mathrm{g} / \mathrm{ml}$ to 430,392 , and $0.35 \mu \mathrm{g} / \mathrm{ml}$ for the Q-Naturale system, and from an initial value of $560 \mu \mathrm{g} / \mathrm{ml}$ to 464,388 , and 0.20 $\mu \mathrm{g} / \mathrm{ml}$ for the WPI system, respectively. These results suggest that storing the nanoemulsions at refrigerator temperatures improves the stability of the encapsulated $\beta$-carotene to degradation for both types of emulsifier. Nevertheless, there did not appear to be major differences in the ability of the two natural emulsifiers to inhibit lipid oxidation, since the loss of $\beta$-carotene over time was fairly similar for both systems.

(a)

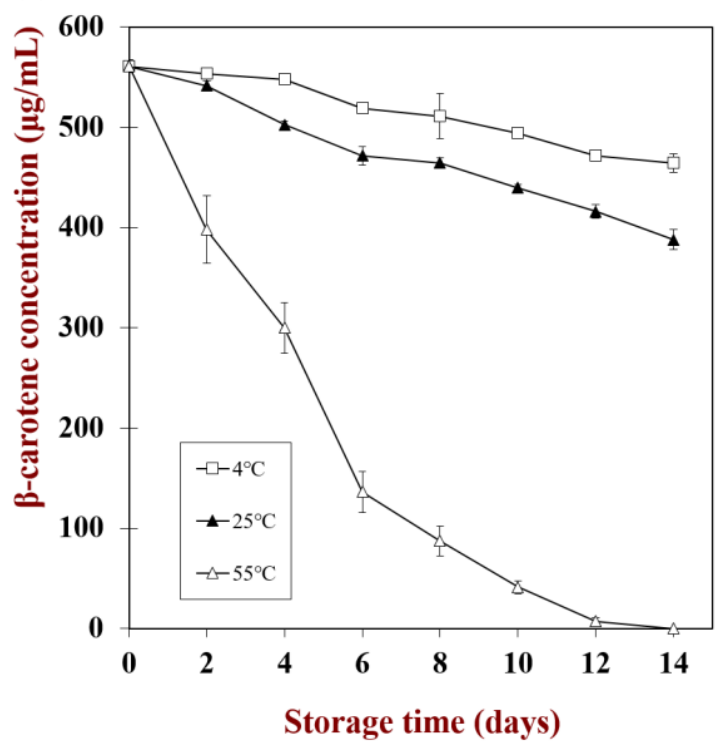

(b)

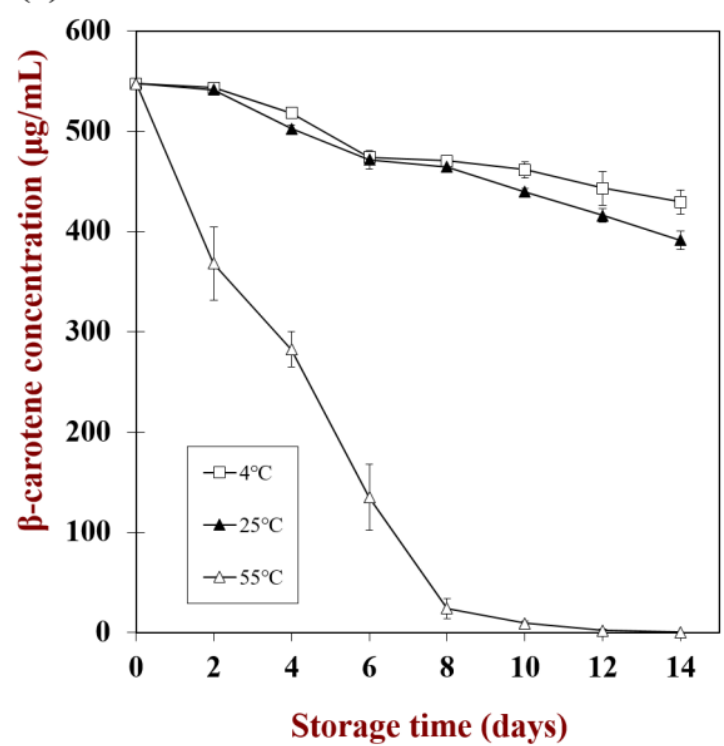

Fig.6. Influence of storage temperature on total concentrations of $\beta$-carotene-loaded nanoemulsions stabilized by (a) Q-Naturale or (b) WPI ( $0.05 \% \beta$-carotene, $10 \%$ cron oil, $0.02 \%$ sodium azide, $5 \mathrm{mM}$ phosphate buffer, $\mathrm{pH} 7)$.

\section{Conclusions}

It was hypothesized that a newly commercialized dual-channel microfluidizer would be suitable for the continuous production of nutraceutical nanoemulsions. This study 
showed that $\beta$-carotene enriched nanoemulsions (diameter $<200 \mathrm{~nm}$ ) stabilized by natural emulsifiers can be fabricated using this approach. The size of the droplets in the nanoemulsions decreased with increasing emulsifier concentration and homogenization pressure, which meant that lipid droplets with different sizes could be produced by tuning operating conditions. $\beta$-carotene-enriched nanoemulsions containing small stable lipid droplets could be formed at an emulsifier-to-oil ratio of 1:10 and an operating pressure of 15 kpsi (103 $\mathrm{MPa})$ when either quillaja saponins or whey proteins were used as emulsifiers. The mean particle diameters were between 140 to $160 \mathrm{~nm}$ and the particle size distributions were monomodal after one pass through the homogenizer. To the authors knowledge, this is the first study to demonstrate that dual-channel microfluidization can be successfully employed to produce nutraceutical-enriched nanoemulsions. The main advantages of this method compared to conventional high-pressure valve homogenizers and single channel microfluidizers is that small droplets can be formed continuously using a single pass through the device, without the need of preparing an emulsion premix [21, 27].

The impact of storage temperature on the physical and chemical stability of $\beta$-carotene enriched nanoemulsions stabilized by either quillaja saponins or whey proteins were then measured. After 14 days, nanoemulsions stabilized by quillaja saponins remained stable to droplet aggregation when stored at 4 or $25{ }^{\circ} \mathrm{C}$, but exhibited a small amount of aggregation when stored at $55{ }^{\circ} \mathrm{C}$. Conversely, the nanoemulsions stabilized by whey proteins remained stable to aggregation at all three incubation temperatures. There was a pronounced decrease in the amount of $\beta$-carotene remaining in the nanoemulsions during storage due to chemical degradation, which led to color fading. The rate of chemical degradation and color fading increased with increasing temperature, with the most rapid change occurring between 25 and $55{ }^{\circ} \mathrm{C}$. The type of natural emulsifier used did not have a major impact on the rate of carotenoid degradation and 
color fading.

Overall, these results have important implications for the rational design of nanoemulsion-based delivery systems to encapsulate, protect, and deliver carotenoids in foods, pharmaceutical, and other commercial products. Future work should focus on developing effective strategies to stabilize the encapsulated carotenoids from chemical degradation in nanoemulsions, e.g., by adding antioxidants or chelating agents, or by controlling interfacial composition or charge $[17,18]$. Nanoemulsions are particularly susceptible to this kind of interfacial chemical degradation due to their large surface areas.

\section{Acknowledgements}

Xiang Luo (CSC No.: 201508210193) would like to thank the Chinese Scholarship Council for support. This material was partly based upon work supported by the Cooperative State Research, Extension, Education Service, USDA, Massachusetts Agricultural Experiment Station (MAS00491) and an USDA, NRI Grant (2013-03795).

\section{References}

[1] A. R. Mangels, J. M. Holden, G. R. Beecher, M. R. Forman, E. Lanza. Carotenoid contents of fruits and vegetables: an evaluation of analytical data. J Am Diet Assoc, 1993, 93:284-96.

[2] E. J. Johnson. The role of carotenoids in human health. Nutr Clin Care, 2002, 5: 47-9.

[3] S. Agarwal, A. V. Rao. Carotenoids and chronic diseases. Drug Metab Drug Interact, 2000,17:189-210.

[4] S. A. Paiva, R. M. Russell. Beta caroteneand other carotenoids as antioxidants. J Am Coll Nutr, 1999, 18, 426-433. 
[5] P. Astrog, S. Gradelet, R. Berges, M. Suschetet. Dietary lycopene decreases initiation of liver preneoplastic foci by diethylnitrosamine in rat. Nutr Cancer, 1997, 29: 60-8.

[6] C. S. Boon, D. J. McClements, J. Weiss, E.A. Decker. Factors influencing the chemical stability of carotenoids in foods. Crit Rev Food Sci Nutr, 2010, 50: 515-532.

[7] J. Rao, E. A. Decker, H. Xiao, D.J. McClements. Nutraceutical nanoemulsions: influence of carrier oil composition (digestible versus indigestible oil) on $\beta$-carotene bioavailability. J Sci Food Agric, 2013, 93: 3175-3183.

[8] L. Mao, D. Xu, J. Yang, F. Yuan, YX Gao, and J. Zhao. Effects of small and large molecule emulsifiers on the characteristics of $\beta$-carotene nanoemulsions prepared by high pressure homogenization. Food Technol Biotech, 2009, 47: 336-342.

[9] C. Qian, E. A. Decker, H. Xiao, D.J. McClements. Physical and chemical stability of $\beta$-carotene-enriched nanoemulsions: Influence of $\mathrm{pH}$, ionic strength, temperature, and emulsifier type. Food Chem, 2012, 132: 1221-1229.

[10] C. Qian, E. A. Decker, H. Xiao, and D.J. McClements. Nanoemulsion delivery systems: Influence of carrier oil on $\beta$-carotene bioaccessibility. Food Chem, 2012, 135: $1440-1447$.

[11] J. W. Erdman, T. L. Bierer, E. T. Gugger. Absorption and transport of carotenoids. Ann NY Acad Sci, 1993, 691: 76-85.

[12] R. S. Parker. Carotenoid and tocopherol composition in human adipose tissue. Am J Clin Nutr 1988, 47: 33-6.

[13] C. Qian, E. A. Decker, H. Xiao, D.J. McClements. Inhibition of $\beta$-carotene degradation in oil-in-water nanoemulsions: Influence of oil-soluble and watersoluble antioxidants. Food Chem, 2012, 135, 1036-1043.

[14] M. van Lieshout, C. E. West, D. Permaesih, Y. Wang, X.Y. Xu, R.B. van Breemen, A.F.L. Creemers, M.A. Verhoeven, and J. Jugtenburg. Bioefficacy of $\beta$-carotene dissolved in oil studied in children in Indonesia. Am J Clin Nutr, 2001, 73: 949-958. 
[15] D. J. McClements. Food Emulsions: Principles, Practice, and Techniques. Boca Raton: CRC Press, 2015.

[16] E. Acosta. Bioavailability of nanoparticles in nutrient and nutraceutical delivery. Curr Opin Colloid Interface Sci, 2009, 14, 3-15.

[17] D. J. McClements, E. A. Decker. Lipid oxidation in oil-in-water emulsions: Impact of molecular environment on chemical reactions in heterogeneous food systems. J Food Sci, 2000, 65: 1270-1282.

[18] T. Waraho, D. J. McClements, E. A. Decker. Mechanisms of lipid oxidation in food dispersions. Trends Food Sci Technol, 2011, 22: 3-13.

[19] M. Hu, D. J. McClements, E. A. Decker. Lipid oxidation in corn oil-in-water emulsions stabilized by casein, whey protein isolate, and soy protein isolate. J Agric Food Chem, 2003, 51: 1696-1700.

[20] S. Mitra, S. R. Dungan. Micellar properties of quillaja saponin.1. Effects of temperature, salt, and pH on solution properties. J Agric Food Chem, 1997, 45, 1587-1595.

[21] L. Bai, D. J. McClements. Development of microfluidization methods for efficient production of concentrated nanoemulsions: Comparison of single-and dual-channel microfluidizers. J Colloid Interface Sci, 2016, 466: 206-212.

[22] R. A. Itle, E. A. Kabelka. Correlation between $\mathrm{L}^{*} \mathrm{a}^{*} \mathrm{~b}^{*}$ color space values and carotenoid content in pumpkins and squash (Cucurbita spp.). HortScience, 2009, 44: 633-637.

[23] R. G. McGuire. Reporting of objective colour measurements. Hortscience, 1992, 27, 1254-1255.

[24] S. M. Jafari, E. Assadpoor, Y.H. He, and B. Bhandari. Re-coalescence of emulsion droplets during high-energy emulsification. Food Hydrocolloids, 2008, 22: 1191-1202. 
[25] K. W. Min, H. I. Gostin. Simulation of Semi-batch emulsion polymerization reactors for polyvinyl chloride (PVC) system. Ind Eng Chem Prod RD, 1979, 18: 272-278.

[26] Y. Zhang, Y. Chen, P. Westerhoff, and J. Crittenden. Impact of natural organic matter and divalent cations on the stability of aqueous nanoparticles. Water Research, 2009, 43: 4249-4257.

[27] L. Lee, I. T. Norton. Comparing droplet breakup for a high-pressure valve homogeniser and a Microfluidizer for the potential production of food-grade nanoemulsions. J. Food Eng., 2013, 114: 158-163.

[28] S. M. Jafari, Y. H. He, B. Bhandari. Nano-emulsion production by sonication and microfluidization-a comparison. Int. J. Food Prop., 2006, 9: 475-485.

[29] T.J. Wooster, D. Labbett, P. Sanguansri, and H. Andrews. Impact of microemulsion inspired approaches on the formation and destabilisation mechanisms of triglyceride nanoemulsions. Soft Matter, 2016, 12: 1425-1435.

[30] P. Walstra. Principles of emulsion formation. Chem. Eng. Sci., 1993, 48: 333.

[31] P. Walstra. Physical chemistry of foods. New York, NY: 2003, Marcel Decker.

[32] C. Freitas, R. H. Müller. Effect of light and temperature on zeta potential and physical stability in solid lipid nanoparticle (SLN ${ }^{\mathrm{TM}}$ ) dispersions. Int J Pharm, 1998, 168: 221-229.

[33] I. Roland, G. Piel, L. Delattre, and B. Evrard. Systematic characterization of oil-in-water emulsions for formulation design. Int J Pharm, 2003, 263: 85-94. 


\section{Graphical Abstract}

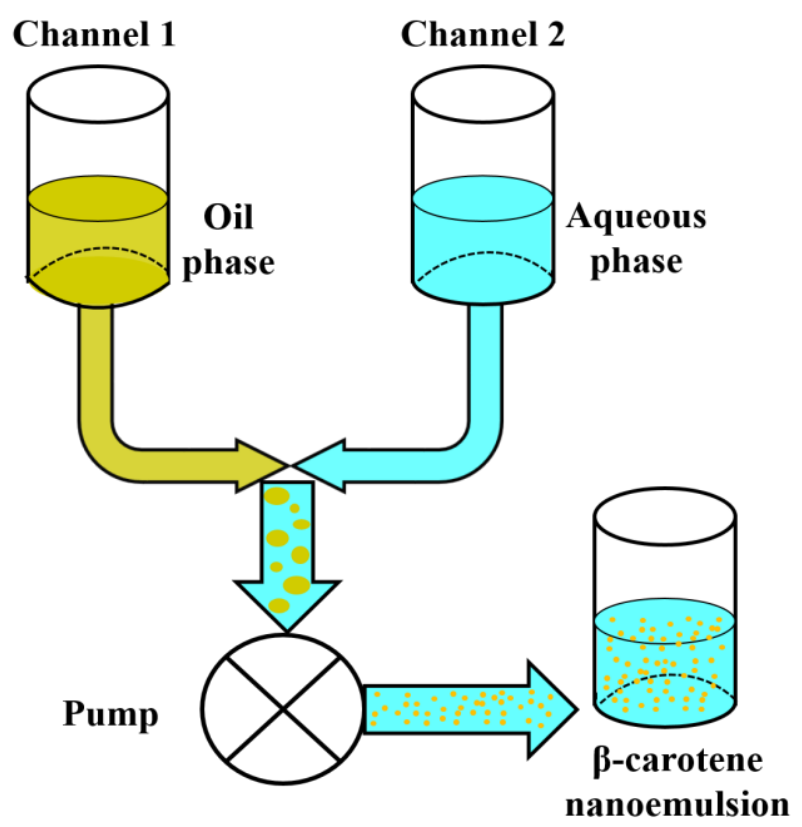

